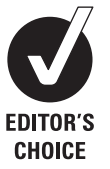

CHOICE

\title{
Sclerosing polidocanol injections or arthroscopic shaving to treat patellar tendinopathy/jumper's knee? A randomised controlled study
}

\author{
Lotta Willberg, ${ }^{1} 2$ Kerstin Sunding, ${ }^{1}$ Magnus Forssblad, ${ }^{1}$ Martin Fahlström, ${ }^{3}$ \\ Håkan Alfredson ${ }^{2}$
}

${ }^{1}$ Capio Artro Clinic, Stockholm Sports Trauma Research Centre, Karolinska Institutet, Stockholm, Sweden ${ }^{2}$ Sports Medicine Unit, Department of Surgical and Perioperative Science, University of Umeå, Umeå, Sweden

${ }^{3}$ Department of Community Medicine and Rehabilitation Rehabilitation Medicine, University of Umeå, Umeå Sweden

\section{Correspondence to}

Dr Lotta Willberg, Capio Artro Clinic, Stockholm Sports Trauma Research Centre, Karolinska Institutet, Sophiahemmet, Valhallavägen 91, S-114 86 Stockholm, Sweden:

lotta.willberg@capio.se

Accepted 3 February 2011

\begin{abstract}
Background Proximal patellar tendinopathy/jumper's knee (PT/JK) is well known to be difficult to treat. Recent studies using an ultrasound and colour Dopplerbased treatment approach on the dorsal side of the tendon, sclerosing polidocanol injections and ultrasoundguided arthroscopic shaving, have shown promising clinical results.
\end{abstract}

Objectives To compare the clinical effects after treatment with sclerosing polidocanol injections and arthroscopic shaving.

Material and methods 52 patellar tendons (43 men and two women) with ultrasound and colour Dopplerverified diagnosis of PT/JK were randomly assigned to treatment with ultrasound and colour Doppler-guided sclerosing polidocanol injections (group A) or ultrasound and colour Doppler-guided arthroscopic shaving (group B). All patients were involved in patellar tendon loading sports or recreational activities, and had had a long duration of pain symptoms from the proximal patellar tendon. Pain during patellar tendon loading activity, and at rest, before and after treatment (visual analogue scale; VAS), and patient satisfaction with the result of the treatment, was registered.

Results After treatment, the patients treated with arthroscopic shaving had a significantly lower VAS score at rest and during activity, and were significantly more satisfied compared with the patients in the sclerosing injection group.

Conclusions Both treatment with ultrasound and colour Doppler-guided sclerosing polidocanol injections and arthroscopic shaving showed good clinical results, but patients treated with arthroscopic shaving had less pain and were more satisfied with the treatment result. Because surgical treatment is a one-stage treatment return to sports was faster in this group.

Chronic patellar tendon pain (patellar tendinopathy (PT)/jumper's knee (JK)) is a most often painful condition with unknown aetiology and pathogenesis, known to be troublesome to treat. ${ }^{1-3}$

Conservative treatment of chronic patellar tendon pain, $\mathrm{PT} / \mathrm{JK}$ using painful eccentric quadriceps training has shown some good results, ${ }^{4-6}$ but among athletes involved in jumping sports this treatment has been less successful. Traditional surgical treatment most often includes open patellar tenotomy and excision of the region with tendon changes. Sometimes ultrasound-guided percutaneous longitudinal tenotomy, curettage, multiple drilling of the inferior patellar pole, or excision of the distal patellar tip, is used. ${ }^{7-12}$ After these treatments there is a relatively long rehabilitation period. The clinical results of surgery have been shown to be varying and often poor. ${ }^{13}$ A recent randomised study comparing treatment with eccentric quadriceps training and traditional open tenotomy plus excision showed similar, but only $50-60 \%$ good clinical results in the groups. ${ }^{14}$

During the past few years it has been debated where the pain comes from in this and other chronic painful tendinopathies. ${ }^{15}$ Recent studies using ultrasound and colour Doppler and immunohistochemical analyses of tendon biopsies, have shown high blood flow ${ }^{16} 17$ and nerves ${ }^{18} 19$ on the dorsal side of the proximal patellar tendon. These findings have led to research on new treatment methods, sclerosing polidocanol injections ${ }^{20}$ 21 and ultrasound-guided arthroscopic shaving, ${ }^{22}$ targeting the treatment outside the dorsal patellar tendon, ie, where the high blood flow and nerves have been demonstrated.

The aims of this randomised study were to compare the clinical results after treatment with ultrasound and colour Doppler-guided sclerosing polidocanol injections and ultrasound-guided arthroscopic shaving to try to clarify whether either treatment, both performed outside the tendon, is significantly superior to the other.

The primary outcome measure was to evaluate the clinical effect of the treatment by having the patients to score the level of patellar tendon pain during their specific sport or recreational activity, and at rest, and evaluate patient satisfaction with the results of the treatment.

\section{MATERIAL AND METHODS Patients}

Forty-five patients (52 tendons) were included in the study. All were patients referred to the Capio Artro Clinic in Stockholm, with the diagnosis PT/ $\mathrm{JK}$. The diagnosis was confirmed with ultrasound and colour Doppler showing structural tendon changes and high blood flow on the dorsal side of the proximal patellar tendon. All patients had had a long duration of pain symptoms during patellar tendon loading activity, and seven patients had bilateral tendon changes. No patient had had an acute onset of pain. The patients had tried different types of treatment before referral, such as; rest for more than 3 months ( $n=45)$, eccentric training $(n=33)$ and non-steroidal anti-inflammatory drugs 
$(\mathrm{n}=11)$. All patients were active, ranging from recreational $(n=19)$ to competition level $(n=26)$.

All patients were diagnosed (clinically and by ultrasound and colour Doppler examination) before inclusion to have chronic painful $\mathrm{PT} / \mathrm{JK}$ in the proximal patellar tendon. All tendons were examined with high resolution grey-scale ultrasound and with colour Doppler (Acuson Antares Sonoline, Capio Artro Clinic, Stockholm, Sweden). A WSX 13-5 linear multifrequency (7-11.4 MHz) probe was used. The pathological changes in the thickened proximal patellar tendon were recorded (figure 1). Colour Doppler was used to diagnose neovascularisation/high blood flow, and to locate where the vessels entered the tendon. The contralateral tendon was also examined.

After randomisation there were 24 men (24 tendons) and two women (two tendons), mean age 26 years (range 17-36), and a long duration (mean 20 months, range 8-60 months) of pain symptoms in group A (treatment with sclerosing polidocanol injections). In group B (treatment with arthroscopic shaving) there were 25 men (25 tendons) and one woman (one tendon), mean age 26 years (range 16-38), and a long duration (mean 24 months, range 6-60 months) of pain symptoms. For more patient characteristics, see table 1 .

\section{Interventions}

Two different treatments were used, ultrasound plus Dopplerguided sclerosing polidocanol injections ${ }^{20}(10 \mathrm{mg} / \mathrm{ml})$ and ultrasound plus Doppler-guided arthroscopic shaving. ${ }^{22}$
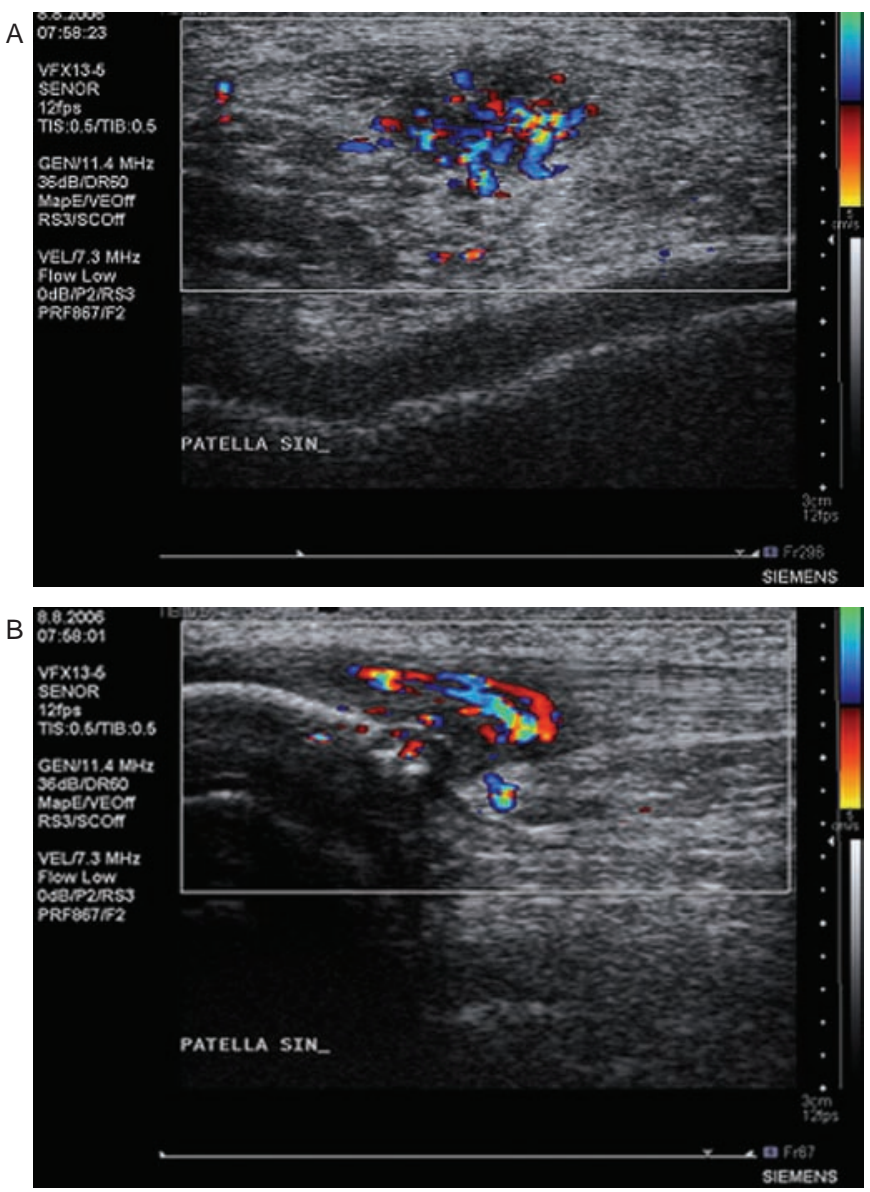

Figure 1 Grey-scale ultrasound and colour Doppler scan of a patient with chronic painful patellar tendinopathy/jumper's knee. (A) Longitudinal and (B) cross-sectional plane. Tendon thickening, structural tendon changes, and hypo-echoicity in the dorsal tendon.
Sclerosing polidocanol injections

The injection was performed with a $0.7 \times 50 \mathrm{~mm}$ needle connected to a $2 \mathrm{ml}$ syringe. The same experienced ultrasonographer performed all ultrasound and colour Doppler examinations and injections. The injection was performed dynamically, with the aid of real-time grey-scale ultrasound and the colour Doppler technique, to inject at the target vessels. Small volumes $(0.1-0.2 \mathrm{ml}$, maximum $2 \mathrm{ml})$ of the substance were injected into the regions with local neovascularisation/high blood flow dorsal to the proximal patellar tendon. A dressing was applied for $24 \mathrm{~h}$. A maximum of three injection treatments (at least 6 weeks in between) were given before evaluation.

\section{Arthroscopic shaving}

Arthroscopy was performed under local anaesthesia. The patients were in a supine position with straight knee and relaxed quadriceps. We used standard anteromedial and anterolateral portals, and a pressure controlled pump. No tourniquet was used. Ultrasound and colour Doppler was used preoperatively. Initially, a standard arthroscopic evaluation of the whole knee joint was performed. Then the patellar tendon insertion into the patella was identified. For shaving, a $4.5-\mathrm{mm}$ full radius blade shaver was used. Simultaneous ultrasound examination (longitudinal and transversal views) guided the procedure. Careful shaving, aiming to destroy only the region with high blood flow (neovessels) and nerves adjacent to the tendinosis changes on the dorsal side of the tendon, was done (ie, separating the Hoffa fat pad from the patellar tendon). No tendon tissue was resected and the Hoffa fat pad was saved. The portals were closed with a tape, and a bandage was used for $24 \mathrm{~h}$.

\section{After treatment}

The patients in both groups were allowed full weightbearing walking immediately after the treatment. Two weeks after treatment the patients were told gradually to increase the patellar tendon load up to full loading. There was no specific rehabilitation protocol, or set time frames, preceding full tendon loading activity.

\section{Follow-ups}

After treatment with sclerosing polidocanol injections the patients were followed up at 6-8 weeks after each treatment. If there was remaining tendon pain during sport activity, and remaining high blood flow in the region with structural tendon changes at follow-up, another injection treatment was given.

After treatment with arthroscopic shaving the patients were followed up at 2 weeks and at 6-8 weeks postoperatively.

Further follow-ups were done at 6 and 12 months after treatment.

\section{Outcomes}

The patients scored the level of patellar tendon pain during their specific sport activity, and at rest, on a $100-\mathrm{mm}$ long visual analogue scale (VAS). The amount of pain was recorded from 0 to $100 \mathrm{~mm}$, where no pain was recorded as 0 and severe pain as 100 .

Self-reported patient satisfaction with the result of the treatment (0-100\% satisfaction), was also scored by the patients on a 100-mm long scale. 
Table 1 Basic data and treatment outcome for group A and group B

\begin{tabular}{|c|c|c|c|}
\hline & Group A - sclerosing injections & Group B - arthroscopy & \\
\hline Variable & Mean (SD) & Mean (SD) & Significance ( $p$ value) \\
\hline Age, years & $27.0(7.6)$ & $26.6(7.6)$ & 0.755 \\
\hline Duration of symptoms, months & $20.0(10.4)$ & $23.8(15.5)$ & 0.596 \\
\hline VAS at rest at assessment & $37.8(24.9)$ & $44.6(28.4)$ & 0.431 \\
\hline VAS at activity at assessment & $69.0(17.3)$ & $76.5(13.6)$ & 0.143 \\
\hline Follow-up time, months & $13.7(6.9)$ & $12.9(7.8)$ & 0.719 \\
\hline VAS at rest at follow-up & $19.2(23.2)$ & $5.0(8.3)$ & 0.004 \\
\hline VAS at activity at follow-up & $41.1(28.5)$ & $12.8(19.3)$ & 0.001 \\
\hline Satisfaction with result, 0-100 & $52.9(32.6)$ & $86.8(20.8)$ & 0.000 \\
\hline
\end{tabular}

VAS, visual analogue scale.

A

Group A, sclerosing injections

\section{VAS during activity before intervention (1) and at follow-up (2)}

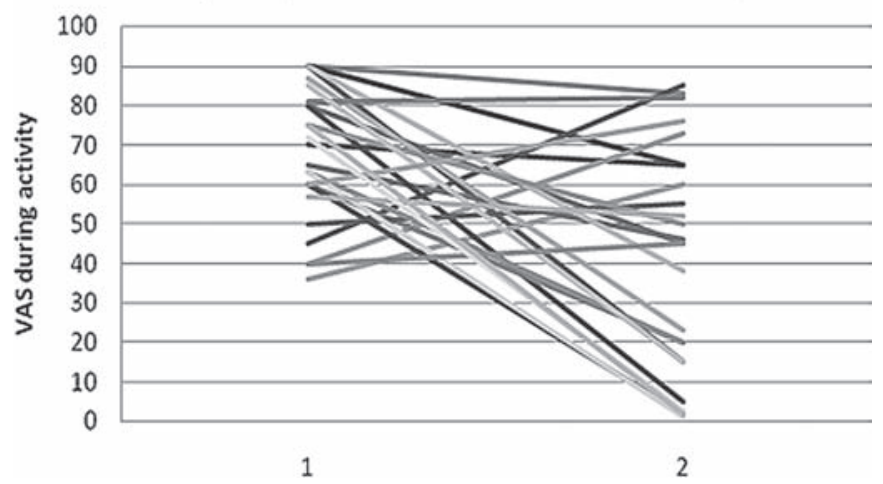

B VAS during activity before intervention (1) and at follow-up (2)

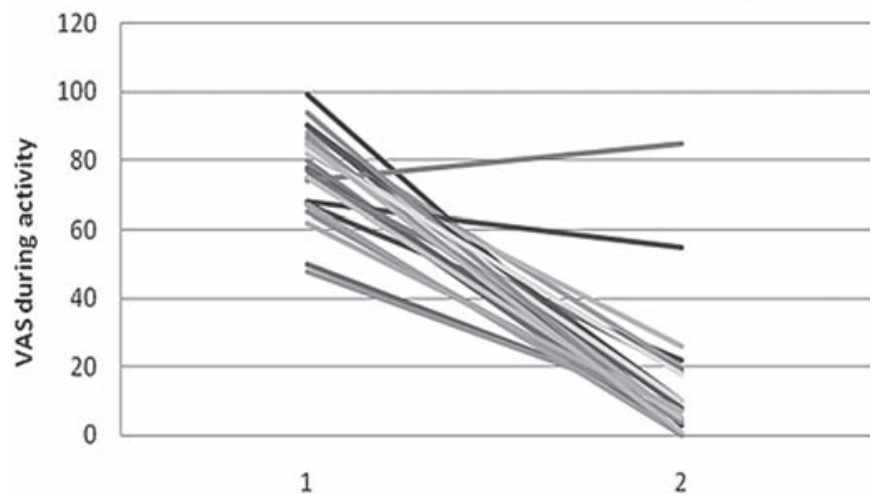

Figure 2 (A) The results in group A, presented as visual analogue scale (VAS) during activity before and after treatment. (B) The results in group $B$, presented as VAS during activity before and after treatment.

\section{Sample size/power analysis}

The power analysis was based on the results from two previous pilot studies. ${ }^{20} 22$ The analysis showed that 20 individuals in each group were needed to give a power of $80 \%$ to find a difference of $50 \mathrm{~mm}$ in VAS between the groups, on a $5 \%$ significance level. We estimated a higher difference than normally accepted (a difference in VAS of $30 \mathrm{~mm}$ ), to make sure that the groups were not too small.

\section{Randomisation}

After receiving oral and written information, from an independent assistant the patients selected an envelope (52 opaque envelopes), allocating themselves to either treatment with sclerosing polidocanol injections $(10 \mathrm{mg} / \mathrm{ml})$ or treatment with arthroscopic shaving.

The outcomes of the treatments were self-assessed by the patients. The assistant who collected the outcome measures was blinded to the group allocation when the patients scored their VAS and satisfaction with treatment.

\section{Statistical evaluation}

The SPSS package (version 18.0) was used for all statistical calculations. Mean and SD were used to describe data. Differences between groups were calculated using a non-parametric test for independent samples (Mann-Whitney U test). A p value of less than 0.05 was considered statistically significant.

\section{RESULTS}

In group $\mathrm{A}$, one patient did not continue the study because of pregnancy. In group B, all but one patient received the intended treatment.

Before treatment, there were no significant differences between the treatment groups with regard to age, duration of symptoms and pain at rest or during patellar tendon loading activity.

After treatment, the patients treated with arthroscopic shaving had significantly lower VAS scores for pain at rest and during patellar tendon loading activity, and were significantly more satisfied with the treatment result, compared with the patients treated with sclerosing polidocanol injections.

The results are shown in figure 2 .

\section{DISCUSSION}

In this randomised study on patients with chronic painful proximal PT/JK, comparing treatment with sclerosing polidocanol injections and arthroscopic shaving, we found that the patients treated with arthroscopic shaving had less pain and were more satisfied with the treatment result than patients treated with sclerosing polidocanol injections.

We used ultrasound guidance for both sclerosing polidocanol injections and arthroscopic shaving. Ultrasound is a reliable method to evaluate tendon structure and thickness, ${ }^{17}$ and is well suited to guide procedures in which optimal precision is needed. For sclerosing polidocanol injections we used ultrasound and colour Doppler guidance to find the target region (region with high blood flow outside the structurally abnormal dorsal tendon), but for arthroscopic shaving only ultrasound was used. This is because the epinephrine in the local anaesthetic, together with the fluid pressure in the knee joint, decreases the blood flow and thereby reduces the possibility of registering high blood flows. However, ultrasound plus colour Doppler was used for the preoperative planning of the procedure. 
The information in this study refers mainly to men, in contrast to other studies on patients with PT/JK. However, the material consists of consecutive patients from the Stockholm region referred to the Capio Artro clinic with this diagnosis, and we therefore believe it is representative of the situation in this region. Maybe there are regional differences depending on the popularity of certain sports? In countries such as Norway, where sports such as handball are very popular among women, it is likely that there will be more women with PT/JK. ${ }^{21}$

Treatment of PT/JK has for many years puzzled clinicians, and the background to pain has never clearly been resolved in research. Vague knowledge about where the pain comes from, and varying clinical results with the different treatment methods in use, have raised many questions. However, recent histology findings, in which nerves were found in close relation to blood vessels on the dorsal side of the proximal patellar tendon, ${ }^{23}$ have been shown to be possible targets for treatment. Pilot studies using both ultrasound plus colour Dopplerguided sclerosing polidocanol injections and ultrasound plus colour Doppler-guided arthroscopic shaving, targeting the region with high blood flow and nerves outside the dorsal tendon, have been shown to reduce tendon pain significantly. ${ }^{20-22}$ The results of those studies, together with the current study, strengthen the likelihood that the source of pain in $\mathrm{PT} / \mathrm{JK}$ is on the dorsal side of the tendon. Treatment outside the dorsal tendon alone is in strong contrast to the method used worldwide in which there is intratendinous excision of macroscopically abnormal tendon tissue through a longitudinal tenotomy. It is interesting that ultrasound-guided treatment outside the dorsal tendon seems to produce markedly better clinical results than have been reported from intratendinous surgery. ${ }^{14}$ Also, considering the rehabilitation after treatment, extratendinous procedures ${ }^{20-22}$ are associated with a very quick (6-8 weeks) return to full tendon loading activity, whereas intratendinous procedures ${ }^{10} 1214$ often require a very long (4-6 months) rehabilitation period. The current study only presents short-term results, but it seems promising that there is now a minimally invasive surgical treatment method, with no need for a long rehabilitation period, which has the potential to reduce tendon pain significantly.

We did not use a specific rehabilitation protocol after instituted treatment, and allowed patients to return to full patellar tendon loading activity after just 2 weeks. There were no complications among the patients in the current study, but whether a quick return to full activity is the best way to proceed should be discussed. After sclerosing polidocanol injections it has in previous studies been shown that a quick return to full tendon loading activity can be achieved without complications. ${ }^{20} 21$ However, after an arthroscopy, maybe there should be specific rehabilitation to regain thigh muscle function before returning to full activity? Further studies will evaluate whether a specific rehabilitation protocol has an effect on the time to return to previous activity.

In this study, there was a quicker return to sports with minimal or no pain after treatment with the one-stage ultrasoundguided arthroscopic shaving procedure, compared with the patients who were treated with multiple sclerosing polidocanol injections. The long-term outcome ( $>1$ year) will be studied in another follow-up study also including tendon structure and thickness.

It might be considered a limitation in this study that we did not use a specific activity or functional score such as the VISA score (Victorian Institute of Sport Assessment), but because many of the patients involved in the current study were

\section{What is already known on this topic}

$\mathrm{PT} / \mathrm{JK}$ is well known to be a difficult condition to treat.

- Intratendinous surgical treatment is associated with a long rehabilitation period and often poor clinical results.

\section{What this study adds}

- A new surgical approach in which ultrasound and Dopplerguided arthroscopic shaving is performed outside the tendon.

- The method is associated with a relatively short rehabilitation period and a fast return to sports, with good short-term clinical results.

- The method is based on ultrasound and Doppler findings and immunohistochemical analyses of biopsies.

recreational athletes not involved in team training, or had regular training habits, our decision was that the VISA score was less suitable for this patient group. Instead, we focused on evaluating patellar tendon pain during their specific recreational or sport activity and at rest. We believe this, together with satisfaction with the treatment results, are appropriate tools to evaluate the outcome of the treatments.

In conclusion, both treatment with ultrasound and colour Doppler-guided sclerosing polidocanol injections and arthroscopic shaving showed good clinical results, but patients treated with arthroscopic shaving had significantly less pain and were more satisfied with the treatment result. Because surgical treatment is a one-stage treatment return to sports was faster in this group.

\section{Competing interests None.}

\section{Patient consent}

Ethics approval This study was conducted with the approval of the Ethical Committee at the Medical Faculty of the Karolinska Institutet, Stockholm.

Provenance and peer review Not commissioned; externally peer reviewed.

\section{REFERENCES}

1. Cook JL, Khan KM, Harcourt PR, et al. A cross sectional study of 100 athletes with jumper's knee managed conservatively and surgically. The Victorian Institute of Sport Tendon Study Group. Br J Sports Med 1997;31:332-6.

2. Khan KM, Maffulli N, Coleman BD, et al. Patellar tendinopathy: some aspects of basic science and clinical management. Br J Sports Med 1998;32:346-55.

3. Kettunen JA, Kvist M, Alanen E, et al. Long-term prognosis for jumper's knee in male athletes. A prospective follow-up study. Am J Sports Med 2002;30:689-92

4. Jonsson P, Alfredson H. Superior results with eccentric compared to concentric quadriceps training in patients with jumper's knee: a prospective randomised study. Br J Sports Med 2005;39:847-50.

5. Purdam CR, Jonsson $\mathrm{P}$, Alfredson $\mathrm{H}$, et al. A pilot study of the eccentric decline squat in the management of painful chronic patellar tendinopathy. Br J Sports Med 2004;38:395-7.

6. Young MA, Cook JL, Purdam CR, et al. Eccentric decline squat protocol offers superior results at 12 months compared with traditional eccentric protocol for patellar tendinopathy in volleyball players. Br J Sports Med 2005;39:102-5.

7. Cook JL, Khan KM. What is the most appropriate treatment for patellar tendinopathy? Br J Sports Med 2001;35:291-4.

8. Karlsson J, Lundin O, Lossing IW, et al. Partial rupture of the patellar ligament. Results after operative treatment. Am J Sports Med 1991;19:403-8.

9. Johnson DP. Arthroscopic surgery for patellar tendonitis. Arthroscopy 1998;14:44.

10. Saillant G, Rolland E, Garcon P, et al. Surgical treatment of patellar tendinitis. A series of 80 cases. J Traumatol Sport 1991;8:114-20. 
11. Testa V, Capasso G, Maffulli N, et al. Ultrasound-guided percutaneous longitudinal tenotomy for the management of patellar tendinopathy. Med Sci Sports Exerc 1999;31:1509-15.

12. Raatikainen T, Karpakka J, Puranen J, et al. Operative treatment of partial rupture of the patellar ligament. A study of 138 cases. Int J Sports Med 1994;15:46-9.

13. Coleman BD, Khan KM, Maffulli N, et al. Studies of surgical outcome after patellar tendinopathy: clinical significance of methodological deficiencies and guidelines for future studies. Victorian Institute of Sport Tendon Study Group. Scand J Med Sci Sports 2000;10:2-11.

14. Bahr R, Fossan B, Løken S, et al. Surgical treatment compared with eccentric training for patellar tendinopathy (Jumper's knee). A randomized, controlled trial. J Bone Joint Surg Am 2006;88:1689-98.

15. Khan KM, Cook JL, Maffulli N, et al. Where is the pain coming from in tendinopathy? It may be biochemical, not only structural, in origin. Br J Sports Med 2000;34:81-3.

16. Ohberg L, Lorentzon R, Alfredson H. Neovascularisation in Achilles tendons with painful tendinosis but not in normal tendons: an ultrasonographic investigation. Knee Surg Sports Traumatol Arthrosc 2001;9:233-8.

17. Weinberg EP, Adams MJ, Hollenberg GM. Color Doppler sonography of patellar tendinosis. AJR Am J Roentgenol 1998;171:743-4.
18. Forsgren S, Danielson P, Alfredson H. Vascular NK-1 receptor occurrence in normal and chronic painful Achilles and patellar tendons: studies on chemically unfixed as well as fixed specimens. Regul Pept 2005;126:173-81.

19. Danielson P, Andersson G, Alfredson H, et al. Marked sympathetic component in the perivascular innervation of the dorsal paratendinous tissue of the patellar tendon in arthroscopically treated tendinosis patients. Knee Surg Sports Traumatol Arthrosc 2008;16:621-6.

20. Alfredson H, Ohberg L. Neovascularisation in chronic painful patellar tendinosis - promising results after sclerosing neovessels outside the tendon challenge the need for surgery. Knee Surg Sports Traumatol Arthrosc 2005; 13:74-80.

21. Hoksrud A, Ohberg L, Alfredson H, et al. Ultrasound-guided sclerosis of neovessels in painful chronic patellar tendinopathy: a randomized controlled trial. Am J Sports Med 2006;34:1738-46.

22. Willberg L, Sunding K, Ohberg L, et al. Treatment of jumper's knee: promising short-term results in a pilot study using a new arthroscopic approach based on imaging findings. Knee Surg Sports Traumatol Arthrosc 2007;15:676-81.

23. Alfredson $\mathbf{H}$, Ohberg L, Forsgren $S$. Is vasculo-neural ingrowth the cause of pain in chronic Achilles tendinosis? An investigation using ultrasonography and colour Doppler, immunohistochemistry, and diagnostic injections. Knee Surg Sports Traumatol Arthrosc 2003:11:334-8. 